\title{
High short- and long-term mortality in major non-cardiac surgical patients admitted to intensive care unit
}

\section{Sarah Ekeloef ${ }^{1}$, Lau Caspar Thygesen², Ismail Gögenur ${ }^{1}$}

${ }^{1}$ Department of Surgery, Zealand University Hospital, Denmark

${ }^{2}$ National Institute of Public Health, University of Southern Denmark, Denmark

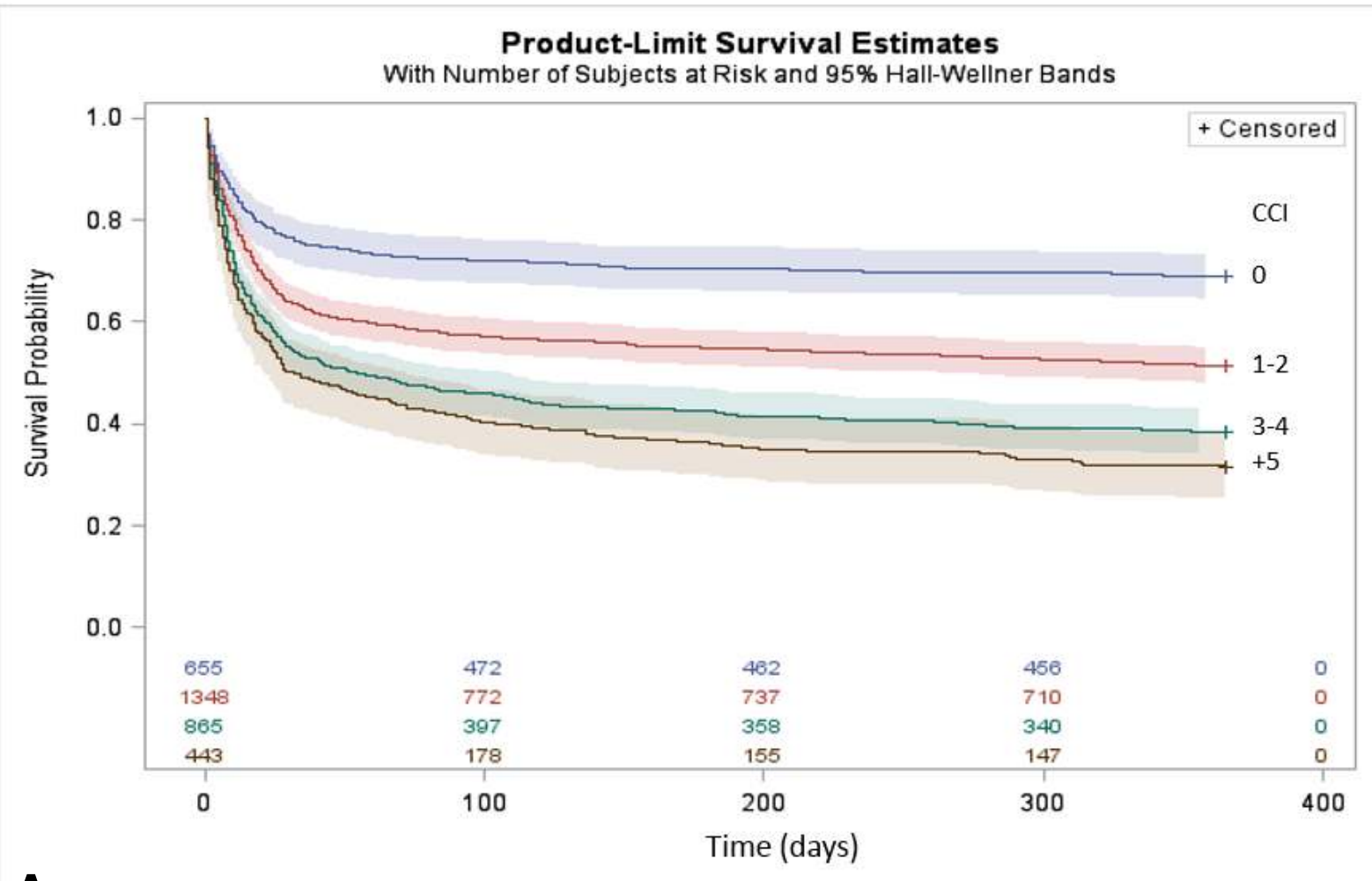

A

Figure 1. A. Kaplan-Meier survival curve stratified by Charlson

Comorbidity Index (CCl). B. Kaplan-Meier survival curve stratified by age.

\section{BACKGROUND AND AIM OF THE STUDY}

Patients undergoing major non-cardiac surgery represent a significant proportion of admissions to intensive care units (ICU) and utilize large amounts of resources. The aim of this study was to present mortality data and identify risk factors associated with 30-days, 90-days and 1-year mortality after major non-cardiac surgery in patients admitted to the ICU.

\section{MATERIALS AND METHODS}

A register based study including patients aged 18+ years admitted to the ICU at four Capital Region hospitals in Denmark between January 2005 and December 2014. Patients were included if admitted to the ICU within 30-days of major surgery with a minimum stay of 24 hours in the ICU unless deceased. Patients were identified through the Danish National Patient Register. Multivariate logistic regression analyses were performed to identify independent risk factors of mortality.

\section{RESULTS AND DISCUSSION}

The study included 3,311 patients admitted to the ICU after undergoing major non-cardiac surgery. Mean age was 69.1 (SD 14.0). Urological surgery accounted for $10.2 \%$, orthopaedic surgery for $18.4 \%$ and abdominal surgery for $71.3 \%$. Acute surgery accounted for $74.5 \%$ of the procedures. Median lengths of stay in the ICU was 2.0 days (1.0-4.0, Q1-Q3). Invasive ventilation was applied in $56.7 \%$ of the patients while $16.2 \%$ received haemodialysis.

The 30-days, 90-days and 1-year mortality was 38\%, 45\% and $51 \%$, respectively. Figure 1 illustrates stratified Kaplan-Meier survival curves. Age, Charlson Comorbidity Index, acute surgery, abdominal and orthopaedic surgery were independently associated with an increased 30-days, 90-days and 1-year postoperative mortality, table 1.

Few studies have specifically reported on outcomes in ICU patients that have undergone major non-cardiac surgery.

Future research should focus on improving the perioperative risk stratification to provide optimal perioperative care and improve the observed poor outcome.

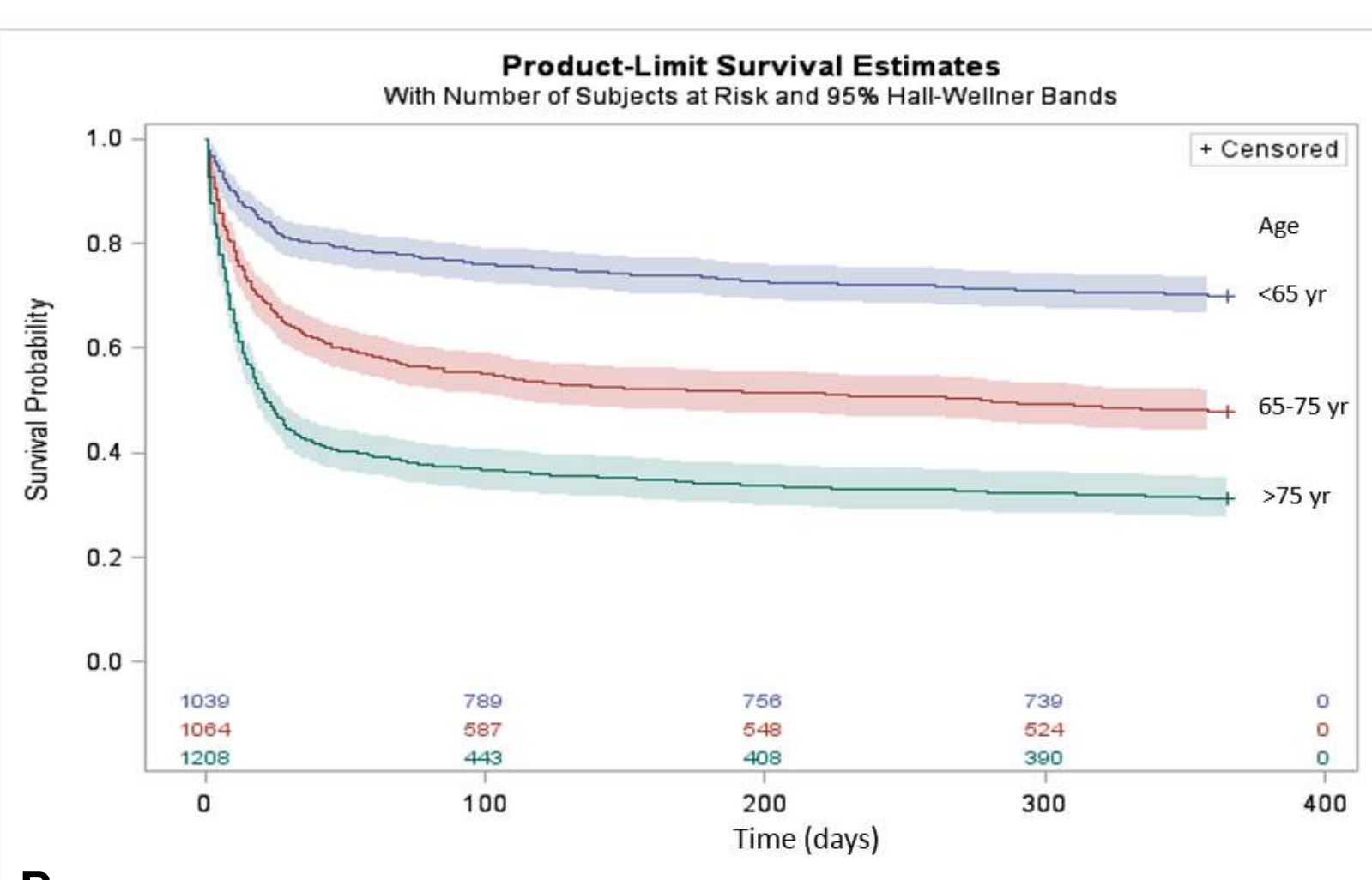

B

Table 1 Multivariate logistic repression models for 30-days, 90-days and 1 year postoperative mortality

\begin{tabular}{|c|c|c|c|c|c|c|}
\hline Covariates & $\begin{array}{l}\text { 30-days } \\
\text { mortality } \\
\text { OR } \\
(95 \% \mathrm{Cl})\end{array}$ & P-value & $\begin{array}{l}90 \text {-days } \\
\text { mortality } \\
\text { OR } \\
(95 \% \text { Cl) }\end{array}$ & P-value & $\begin{array}{l}\text { 1-year } \\
\text { mortality } \\
\text { OR } \\
(95 \% \text { CI) }\end{array}$ & P-value \\
\hline \multicolumn{7}{|l|}{ Sex } \\
\hline Male & 1.0 (REF) & & 1.0 (REF) & & 1.0 (REF) & \\
\hline Female & $1.0(0.8-1.2)$ & 0.82 & $0.90 .8-1.1$ & 0.49 & $0.90 .8-1.0$ & 0.11 \\
\hline Age $(y r)^{*}$ & & $<0.0001$ & & $<0.0001$ & & $<0.0001$ \\
\hline$<65$ & 1.0 (REF) & & 1.0 (REF) & & 1.0 (REF) & \\
\hline $65-75$ & $3.5(2.0-6.1)$ & & $4.2(2.5-7.0)$ & & $3.5(2.2-5.8)$ & \\
\hline$>75$ & $9.7(5.9-15.9)$ & & $\begin{array}{l}10.1(6.3- \\
16.1)\end{array}$ & & $9.5(6.1-14.9)$ & \\
\hline $\begin{array}{l}\text { Charlson } \\
\text { Comorbidity } \\
\text { Index }{ }^{\star \star}\end{array}$ & & $<0.0001$ & & $<0.0001$ & & $<0.0001$ \\
\hline $\mathrm{CCl} O$ & 1.0 (REF) & & 1.0 (REF) & & 1.0 (REF) & \\
\hline CCl 1-2 & $2.4(1.5-3.8)$ & & $2.5(1.6-3.9)$ & & $2.7(1.8-4.0)$ & \\
\hline $\mathrm{CCl} 3-4$ & $4.9(3.0-8.1)$ & & $5.2(3.2-8.3)$ & & $5.1(3.3-7.9)$ & \\
\hline $\mathrm{CCl}+5$ & $6.0(3.4-10.5)$ & & $7.1(4.2-12.1)$ & & $8.6(5.1-14.3)$ & \\
\hline \multicolumn{7}{|l|}{$\begin{array}{l}\text { Surgical } \\
\text { priority }\end{array}$} \\
\hline $\begin{array}{l}\text { Elective } \\
\text { surgery }\end{array}$ & 1.0 (REF) & & 1.0 (REF) & & 1.0 (REF) & \\
\hline $\begin{array}{l}\text { Acute } \\
\text { surgery }\end{array}$ & $2.2(1.8-2.8)$ & $<0.0001$ & $2.1(1.7-2.6)$ & $<0.0001$ & $2.4(2.0-3.0)$ & $<0.0001$ \\
\hline \multicolumn{7}{|l|}{ Cancer } \\
\hline No & 1.0 (REF) & & 1.0 (REF) & & 1.0 (REF) & \\
\hline Yes & $0.9(0.7-1.1)$ & 0.35 & $0.9(0.7-1.2)$ & 0.53 & $1.2(1.0-1.6)$ & 0.08 \\
\hline $\begin{array}{l}\text { Type of } \\
\text { surgery }\end{array}$ & & $<0.0001$ & & $<0.0001$ & & $<0.0001$ \\
\hline Urological & 1.0 (REF) & & 1.0 (REF) & & 1.0 (REF) & \\
\hline Ortopaedic & $4.2(2.7-6.6)$ & & $4.4(2.9-6.8)$ & & $3.2(2.2-4.7)$ & \\
\hline Abdominal & $4.2(2.6-6.8)$ & & $4.1(2.8-6.1)$ & & $2.7(1.9-3.8)$ & \\
\hline
\end{tabular}

*for Charlson Comorbidity Index $0{ }^{* *}$ for age group $<65$ years Significant interaction: age group and Charlson Comorbidity index

$\mathrm{CCl}$, Charlson Comorbidity Index; OR, Odds ratio; 95\%Cl, Walds 95\% confidence interval; REF, reference group

\section{CONCLUSIONS}

Short- and long-term mortality in non-cardiac surgical patients in the ICU remains high especially when admitted after acute surgery. 